\section{Fourier Iterative Method for Induction Logging Interpretation}

\author{
Allen Q. Howard, Jr., Universidade Estadual \\ do Norte Fluminense Darcy Ribeiro
}

Copyright 2014, SBGf - Sociedade Brasileira de Geofísica.

Este texto foi preparado para a apresentação no VI Simpósio Brasileiro de Geofísica, Porto Alegre, 14 a 16 de outubro de 2014. Seu conteúdo foi revisado pelo Comitê Técnico do VI SimBGf, mas não necessariamente representa a opinião da SBGf ou de seus associados. É proibida a reprodução total ou parcial deste material para propósitos comerciais sem prévia autorização da SBGf.

\section{Abstract}

A new method is developed and specialized to solve the electric field integral equation with axial symmetry. The method is applied to induction logging with invasion using only FFT (Fast Fourier Transform) based convolutions and rapidly convergent iterations and is therefore suitable for routine wellsite interpretation. The computational iterative engine requires relatively few lines of code, but in general requires large enough computer memory (at least $32 \mathrm{~Gb}$ ) to fit the relevant 3D arrays in core. Example comparison calculations using the FFT based transforms demonstrate their speed an accuracy. Examples of invasion modeling and comparisons with an analytic radial layered model are given.

\section{Introduction}

Typical integral equation solutions of the induction logging problem often result in full matrices usually limiting such methods to cases where relative anomaly volumes are small. For linearized solutions, the convolutional form of integral equation is often exploited by Fourier transforming equation into spatial frequency domain to perform convolution between the Greens function and volume current using FFTs. For the borehole induction logging problem with invasion however, the integral equation naturally results in order 1 Fourier-Bessel transforms not directly amenable to Cartesian coordinate FFT computation.

Then too, to go beyond linearized form of the integral equation, when for example inter-bed conductivity contrasts are high, iteration is necessary. In this case both forward and inverse Fourier-Bessel transforms of order 1 are required. However, when contrasts are high, iterative methods based upon the Neumann series solution may not converge and the equation needs to be renormalized. A 3D FFT method exploiting underlying axi-symmetry of the fields is developed. Test cases of fields and convolutions computed by conventional and this new method show that it is accurate and significantly faster.

\section{Axial Symmetry Integral Equation}

For an induction tool centered in a circular vertical borehole in a layered environment with invasion, problem becomes axi-symmetric. Then the electric field of transmitter loop has only a $\phi$ component. The first practical and efficient method to compute electromagnetic tool response in such an axi-symmetric formation with layering and invasion appeared in 1984 (Chew et. al.) where good agreement was obtained with a finite element method but was observed to be 250 times faster. The method is sometimes called semi-analytic and was later generalized (Chew et. al. 1993). A higher order approximate solution to axi-symmetric logging problem (Gao, 2006), also uses renormalization (Habashy,1993) and FFT computation. Their efficient method shows good agreement with a more general integral equation method for cases of reasonable step invasion conductivity contrasts and relatively deep invasion. The FFT method here for this geometry requires no such approximations and does not depend on finiteelement radial expansions. Integral equation with axial symmetry in this case is

$$
\mathrm{e}_{\phi}(\mathbf{x})=\mathrm{e}_{\phi}^{(0)}(\mathbf{x})+\frac{1}{2 \pi} \int g_{1}\left(\mathbf{x}, \mathbf{x}^{\prime}\right) j\left(\mathbf{x}^{\prime}\right) \mathrm{d}^{3} x^{\prime},
$$

where the volume current $j(\mathbf{x})$ in terms of profile function $p(\mathrm{x})$ is

$$
\begin{aligned}
& j(\mathbf{x})=p(\mathbf{x}) \mathrm{e}_{\phi}(\mathbf{x}), \\
& p(\mathbf{x})=k^{2}(\mathbf{x})-k_{0}^{2} .
\end{aligned}
$$

Integration in equation (1) is over all-space and the axisymmetric Green's function is

$$
g_{1}\left(\mathbf{x}, \mathbf{x}^{\prime}\right)=\int_{0}^{2 \pi} \cos \left(\phi-\phi^{\prime}\right) g_{0}\left(\mathbf{x}, \mathbf{x}^{\prime}\right) \mathrm{d} \phi^{\prime}
$$

The scalar Green's function $g_{0}\left(\mathbf{x}, \mathbf{x}^{\prime}\right)$ is the familiar

$$
g_{0}\left(\mathbf{x}, \mathbf{x}^{\prime}\right)=\frac{e^{i k_{0} R}}{4 \pi R}, \quad R=\left|\mathbf{x}-\mathbf{x}^{\prime}\right|
$$

Using the convolution theorem in rectangular coordinate $z$, integral equation (1) in spatial-frequency domain $\left(K_{\rho}, K_{z}\right)$ can be shown to have the simple algebraic form

$$
\mathrm{E}\left(K_{\rho}, K_{z}\right)=\mathrm{E}^{(0)}\left(K_{\rho}, K_{z}\right)+\frac{\mathrm{J}\left(K_{\rho}, K_{z}\right)}{K^{2}-k_{0}^{2}} .
$$

In equation (5) transform $\mathrm{J}\left(K_{\rho}, K_{z}\right)$ of the volume current is defined as

$$
\mathrm{J}\left(K_{\rho}, K_{z}\right)=\int_{-\infty}^{\infty} e^{-i K_{z} z} \mathrm{~d} z \int_{0}^{\infty} \rho \mathrm{J}_{1}\left(K_{\rho} \rho\right) j(\rho, z) \mathrm{d} \rho,
$$


where in equation (6) $K^{2}=K_{\rho}^{2}+K_{z}^{2}$ and the unknown total electric field transform $\mathrm{E}\left(K_{\rho}, K_{z}\right)$ is similarly defined.

An important detail is relationship between Fourier-Bessel transforms and 3D Cartesian Fourier transforms. For FFT evaluation of Fourier-Bessel representations such as in equation (6), one approach is to use a generalization of Ferrari's (Ferrari,1995) or Mook's (Mook, 1993) method. Mook determines an equivalence between a FourierHankel transform of order zero and two successive FFTs. This can be generalized to order one transforms as required here, but it is no faster, and is more difficult to implement than simply using 3D Cartesian Fourier transforms. To compute transforms of functions $f(\mathbf{x})$ for typical axi-symmetric loop source excitation, consider Fourier-Bessel transform pair of order $\mathrm{n}, n=0,1,2, \cdots$

$$
\begin{aligned}
F_{n}\left(K_{\rho}\right) & =\int_{0}^{\infty} J_{n}\left(K_{\rho} \rho\right) f_{n}(\rho) \rho \mathrm{d} \rho, \\
f_{n}(\rho) & =\int_{0}^{\infty} J_{n}\left(K_{\rho} \rho\right) F_{n}\left(K_{\rho}\right) K_{\rho} \mathrm{d} K_{\rho} .
\end{aligned}
$$

For order 0 transforms note that since

$$
J_{0}\left(K_{\rho} \rho\right)=\frac{1}{2 \pi} \int_{0}^{2 \pi} e^{-i K_{\rho} \rho \cos \phi} \mathrm{d} \phi,
$$

it follows that Fourier-Bessel transform $F_{0}\left(K_{\rho}\right)$ can also be written as a 2D Cartesian Fourier transform

$$
\begin{aligned}
F_{0}\left(K_{\rho}\right) & =\frac{1}{2 \pi} \int_{0}^{2 \pi} \int_{0}^{\infty} \mathrm{d} \phi \rho \mathrm{d} \rho e^{-i K_{\rho} \rho \cos \phi} f_{0}(\rho), \\
& =\frac{1}{2 \pi} \int_{-\infty}^{\infty} \int_{-\infty}^{\infty} \mathrm{d} x \mathrm{~d} y e^{-i \mathbf{K} \cdot \mathbf{x}} f_{0}\left(\left(x^{2}+y^{2}\right)^{1 / 2}\right),
\end{aligned}
$$

where

$$
\mathbf{K} \cdot \mathbf{x}=K_{x} x+K_{y} y .
$$

Result (9) can be generalized to order one Fourier-Bessel transforms resulting in the pair

$$
\begin{aligned}
F_{1}\left(K_{\rho}\right)= & \frac{i}{2 \pi}\left[\frac{K_{x}}{K_{\rho}} \int \mathrm{d}^{2} x e^{-i \mathbf{K} \cdot \mathbf{x}} \cos \phi f_{1}(\rho)+\right. \\
& \left.\frac{K_{y}}{K_{\rho}} \int \mathrm{d}^{2} x e^{-i \mathbf{K} \cdot \mathbf{x}} \sin \phi f_{1}(\rho)\right], \\
f_{1}(\rho)= & -\frac{i}{2 \pi}\left[\frac{x}{\rho} \int \mathrm{d}^{2} K e^{i \mathbf{K} \cdot \mathbf{x}} \cos \phi_{K} F_{1}\left(K_{\rho}\right)+\right. \\
& \left.\frac{y}{\rho} \int \mathrm{d}^{2} K e^{i \mathbf{K} \cdot \mathbf{x}} \sin \phi_{K} F_{1}\left(K_{\rho}\right)\right] .
\end{aligned}
$$

When formation contrasts are high and or anomaly volumes large, iteration of equation (1) can diverge. Thus for computational reasons, to reduce the norm of the integral operator, use Habashy's (Habashy,1993) normalized form of equation (1), i.e.,

$$
\begin{aligned}
\mathrm{e}_{\phi}(\mathbf{x})= & n(\mathbf{x}) \mathrm{e}_{\phi}^{(0)}(\mathbf{x})+n(\mathbf{x}) \int \tilde{g}_{1}\left(\mathbf{x}, \mathbf{x}^{\prime}\right)\left[\left(k^{2}\left(\mathbf{x}^{\prime}\right)-k_{0}^{2}\right)\right. \\
& \left.\left(\mathrm{e}_{\phi}\left(\mathbf{x}^{\prime}\right)-\mathrm{e}_{\phi}(\mathbf{x})\right)\right] \mathrm{d}^{3} x^{\prime}
\end{aligned}
$$

where the normalization function is defined as

$$
n(\mathbf{x})=[1-m(\mathbf{x})]^{-1},
$$

and where $m(\mathbf{x})$ is defined by the 3D convolution

$$
m(\mathbf{x})=\frac{1}{2 \pi} \int g_{1}\left(\mathbf{x}, \mathbf{x}^{\prime}\right) p(\mathbf{x}) \mathrm{d}^{3} x^{\prime}
$$

Computation of normalization function $m(\mathbf{x})$ provides a good test for FFT based order 1 transforms defined by equations (11) and (12). For radial profiles independent of $z$ note that

$$
P\left(K_{\rho}, K_{z}\right)=2 \pi P\left(K_{\rho}\right) \delta\left(K_{z}\right)
$$

In this case equation (15) takes simple form

$$
m(\mathbf{x})=\int_{0}^{\infty} K_{\rho} \mathrm{d} K_{\rho} J_{1}\left(K_{\rho} \rho\right) \cdot \frac{P\left(K_{\rho}\right)}{K_{\rho}^{2}-k_{0}^{2}}
$$

As a test problem, consider a radial ramp profile is given by

$$
\begin{aligned}
& p(\mathbf{x})=k_{x o}^{2}+[\alpha \rho+\beta]\left(u\left(\rho-r_{0}\right)-u\left(\rho-r_{a}\right)\right)+k_{t}^{2} u\left(\rho-r_{a}\right), \\
& \alpha=\left(k_{t}^{2}-k_{x o}^{2}\right) /\left(r_{a}-r_{0}\right), \\
& \beta=\left(k_{x o}^{2} r_{a}-k_{t}^{2} r_{0}\right) /\left(r_{a}-r_{0}\right),
\end{aligned}
$$

and $u(\rho)$ is the unit step function

$$
u(\rho)= \begin{cases}1, & \rho>0 \\ 0, & \text { otherwise }\end{cases}
$$

Here quasi-static squared wavenumber is $k^{2}=i \omega \mu_{0} \sigma$. Example computations use following parameters. Flushed zone and formation conductivities are $\sigma_{x o}=0.2[\mathrm{~S} / \mathrm{m}]$ and $\sigma_{t}=0.1[\mathrm{~S} / \mathrm{m}]$. Invasion radii are $r_{0}=0.4[\mathrm{~m}]$ and $r_{a}=0.8$ [m]. Borehole radius is $b=0.3048$ [m], with borehole conductivity of $\sigma_{b}=1[\mathrm{~S} / \mathrm{m}]$. Transmitter loop has peak cw current $I_{0}=1[\mathrm{~A}]$, frequency $f=20[\mathrm{kHz}]$, and is located at origin. Loop radius is $a_{T}=0.04$ [m] and has $n_{T}=10$ turns.

For ramp invasion profile, numerical 2D FFT transform of equation (12) is used to compute normalization function $m(\mathbf{x})$ using spectral representation of equation (17). For comparison, function is also computed using a form of representation given by equation (15) as well as another method using known closed form expressions of the profile spectrum. Real and imaginary parts of this function are shown in Fig. 1. The comparison is seen to be excellent.

To test iterative form of renormalized equation defined by equation (13), use ramp profile function given by equation (18) and compute forward and inverse 3D FFT transforms as necessary for one iteration. For test case 512 points 
are used in $x$ and $y$ directions and 256 points in the $z$ direction. This results in large problem of 138 million complex unknown discrete electric field amplitudes. One iteration consisting of a forward and inverse 3D transform takes approximately 7.5 seconds on an i laptop computer. Memory for this computation is approximately $20 \mathrm{~Gb}$.

Fig. 2 compares in amplitude and phase of secondary electric field for first order Born, sixth order Born, and an analytic radial model consisting of $\mathrm{N}$ discrete radial shells. Comparison is versus $\rho$ with $z=-0.368$ [m]. Fig. 3 is the same input parameter comparison for total electric field. Note that zeroth order Born does not at all characterize the anomaly, but as is known, does ok in the total field comparison. Note near transmitter the nonlinear interaction is quite well resolved by sixth order Born. Note also total field magnitude is approximately two orders of magnitude larger than secondary field. Comparison could be better, but the limit of my computer memory permits only a $\Delta x=$ 0.123 [m] discretization used in this example.
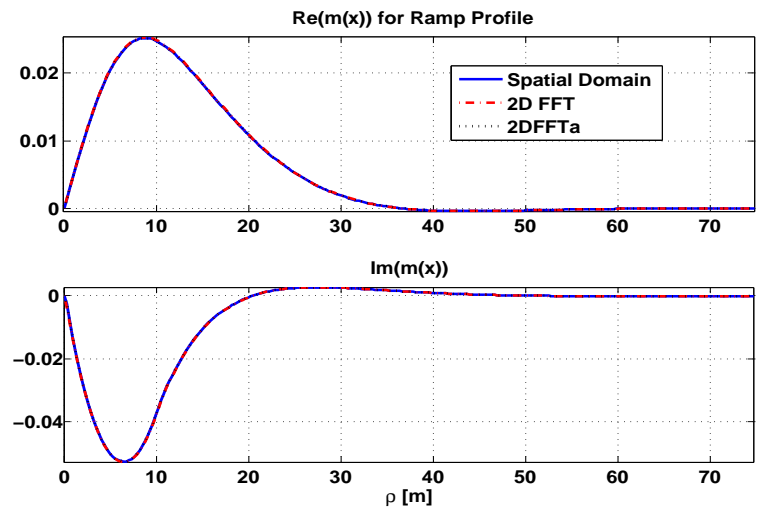

Figure 1: Normalization function defined by equation (15) for ramp profile.

\section{Conclusion}

A new method to solve electric field integral equation has been applied to induction logging problem. Results show promise to compute large scale models in relatively little time. This is important particularly for inverse problem in petrophysical interpretation. An ultimate goal of this research is $3 \mathrm{D}$ vector electromagnetic modeling of tri-axial induction tools.

\section{References}

Chew, W. C., S. Barone, B. Anderson, and C. Hennesy, Diffraction of axi symmetric waves in a borehole by bed boundary discontinuities, Geophysics, vol 49, no. 10, 1984.

Chew, W. C., Z. Nie, Q-H. Liu and B. Anderson, An efficient solution for the response of electrical well logging tools in a complex environment, IEEE Trans. on Geoscience and Remote Sensing, vol 29, no. 2, 1991.

Gao, G. and C. Torres-Verdín, Efficient Numerical Simulation of Axisymmetric Electromagnetic Induction Measurements Using a High-Order Generalized Extended Born Approximation, IEEE Transactions on Geoscience and Remote Sensing, vol 44, no 9, 2006.

Habashy, T. M., R. A. Groom and B. R. Spies, Beyond the Born and Rytov Approximations: A Nonlinear Approach to Electromagnetic Scattering, vol 98, no 82, 1993.
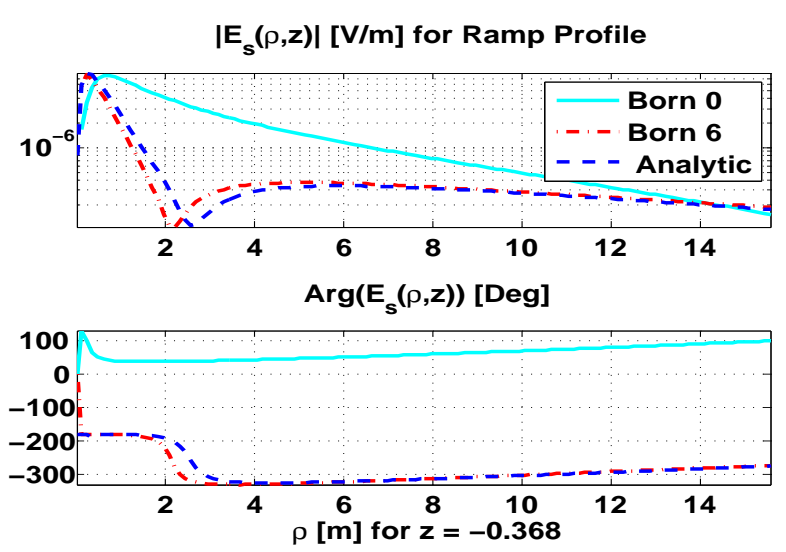

Figure 2: Secondary Electric field comparison as a function of $\rho$.

$\left|E_{\phi}^{(t)}(x)\right|$ for Ramp Profile
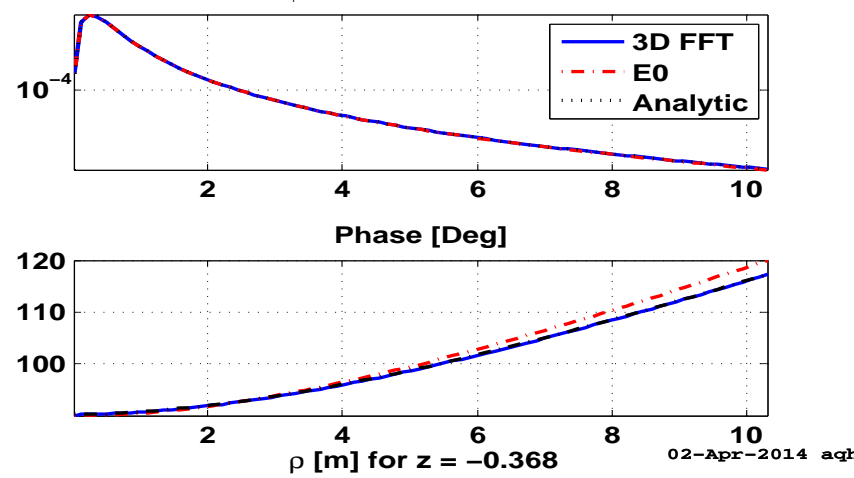

Figure 3: Total Electric field comparison as a function of $\rho$.

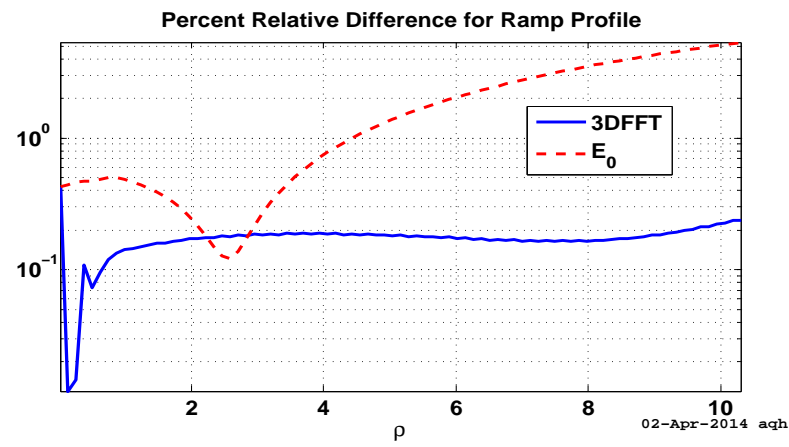

Figure 4: Percent relative difference between total field analytic solution and FFT iteration method.

J. A. Ferrari, Fast Hankel transform of order zero, JOSA Communications, vol. 12, no. 8, 1995.

D. R. Mook, An algorithm for the numerical evaluation of the Hankel and Abel Transforms, IEEE Transactions on acoustics, speech, and signal processing, vol 31, no. 4, 1983.

\section{Acknowledgments}

I thank my host, Professor A. Abel G. Carrasquilla at UENF, Macaé, for providing the opportunity and encouragement to participate again in applied physics in Brazil. 